Revue trimestrielle sur l'image géographique et les formes du territoire

\title{
30 ans de publication dans Mappemonde
}

\section{Clarisse Didelon-Loiseau et Laurent Jégou}

\section{OpenEdition \\ Journals}

Édition électronique

URL : http://journals.openedition.org/mappemonde/2009

DOI : 10.4000/mappemonde.2009

ISSN : 1769-7298

\section{Éditeur}

UMR ESPACE

\section{Référence électronique}

Clarisse Didelon-Loiseau et Laurent Jégou, « 30 ans de publication dans Mappemonde », Mappemonde [En ligne], 127 | 2019, mis en ligne le 01 juillet 2019, consulté le 25 septembre 2020. URL : http:// journals.openedition.org/mappemonde/2009; DOI : https://doi.org/10.4000/mappemonde.2009

Ce document a été généré automatiquement le 25 septembre 2020.

\section{cc) (i) (2) (-}

La revue Mappemonde est mise à disposition selon les termes de la Licence Creative Commons Attribution - Pas d'Utilisation Commerciale - Partage dans les Mêmes Conditions 4.0 International. 


\title{
30 ans de publication dans Mappemonde
}

\author{
Clarisse Didelon-Loiseau et Laurent Jégou
}

1 En juin 2018, nous avons organisé une journée d'études à l'université Paris 1 PanthéonSorbonne pour fêter, avec des lecteurs et des membres passés et présents du comité de rédaction, les 30 ans de publication de la revue Mappemonde avec quelques mois de retard, le tout premier numéro de la revue ayant été publié en 1986. En cette fin d'année 2019, alors que nous approchons déjà des 34 ans de publication, nous proposons dans ce numéro l'ouverture d'un dossier spécial qui rassemble certaines des communications faites lors de cette journée d'étude, mais qui est également enrichi d'articles produits tout spécialement.

2 Nous demandons à nos lecteurs de considérer avec indulgence ce numéro qui est le résultat d'un exercice, il faut bien le reconnaitre, un tantinet nombriliste et tout à fait endogène: l'analyse de l'histoire de la revue par les membres de son comité de rédaction et par ses auteurs. Ce dossier a donc une tournure un peu étrange, mais tout à fait assumée. L'exercice fut également particulier pour les auteurs. En effet, si ces articles n'ont pas l'ambition scientifique que l'on attend normalement des articles publiés dans notre revue ils ont toutefois été produits par leurs auteurs dans les règles de l'art. De même, si le processus d'évaluation n'a pu, exceptionnellement, complètement respecter le sacro-saint processus d'évaluation en double aveugle, chaque article a été évalué par au moins deux, souvent plus, membres du comité de rédaction et ceci avec la même conscience professionnelle que pour des articles suivant une voie plus canonique. Les articles proposés dans ce dossier « 30 ans de publication » sont donc majoritairement écrits par des membres du comité de rédaction, en tant qu'exercice réflexif sur les publications de la revue au cours de ces trente dernières années. Ces textes sont conçus à la fois comme un hommage à nos auteurs et un « cadeau d'anniversaire » à nos lecteurs.

3 Au sein du comité de rédaction, l'exercice, pour artificiel qu'il soit, a eu son utilité : il a été un moment important de passage de témoin entre deux générations du comité. Ce dernier, avec le départ en retraite et l'avancée en âge de membres parfois présents 
depuis les tout débuts de la revue, a fortement été renouvelé ces derniers mois. Il ne compte ainsi presque plus de membres fondateurs ou de personnes présentes à l'origine. Le comité rassemble toutefois toujours des «cartographes" et des "thématiciens" à l'image de notre codirection de rédaction et il s'attache à être toujours aussi ouvert vers l'ensemble des champs de la géographie et vers des disciplines faisant usage de l'image géographique, ouvert sur le monde (correspondants) et ouvert surtout sur la géographie enseignée hors de l'université, avec des représentants du secondaire et des classes préparatoires. Cette journée d'étude a également été l'occasion de marquer un moment de renouveau aussi après quelques mois difficiles, dont le symptôme le plus important a été les retards pris dans la publication, suite au déménagement du secrétariat de rédaction de Toulouse à Avignon, et la maladie et le décès de Guérino Silière dont "la patte " graphique et esthétique est si présente dans les anciens numéros et dont nous essayons encore de préserver l'héritage. Le passage à OpenEdition Journals a également constitué un défi, induisant d'apprendre de nouvelles manières de travailler et soulevant des défis techniques que nous n'avions pas soupçonnés.

4 La préparation de la journée d'étude, comme celle de ce dossier spécial, fut riche d'enseignements. Elle a permis à la fois de revenir sur la naissance fougueuse de la revue, fondée par R. Brunet et R. Ferras à Montpellier, sur l'innovation qu'a constitué en 1986 le lancement d'une revue en couleur dédiée à l'image géographique, sur l'évolution des outils, des méthodes, des concepts mêmes de la cartographie. Mais, plus encore, elle a permis de dresser le tableau de l'évolution de la géographie en tant que discipline dans le contexte français, la majorité de nos articles étant proposés par des géographes, des effets de modes dans les thèmes et les concepts. Elle a également permis de prendre la mesure des évolutions dans l'édition scientifique, avec l'institutionnalisation progressive de la revue et son relatif assagissement, ainsi que la pression pour une «normalisation » de l'article scientifique.

Plus particulièrement, la préparation de la journée des « 30 ans » a donné lieu à la construction de deux bases de données pour analyser l'évolution de notre revue.

- D'une part, une base de données textuelle des «mots clefs » et des résumés, construite et analysée par une équipe associant statisticiens, cartographes et chercheurs : G. Le Campion, O. Pissoat, M. Noucher et L. Jégou. L'objectif consistait à tester l'utilisation de techniques «modernes » d'analyse de contenu textuel pour produire des représentations visuelles expressives, décrivant l'évolution dans le temps des thématiques, des objets, des méthodes dans les (964) articles publiés dans la revue entre 1986 et 2017. Si ce test produit des résultats intéressants, il a aussi permis de délimiter plus précisément les limites de ce type d'exercice. Le jeu de données produit est placé en libre accès pour permettre à nos lecteurs de produire leurs propres représentations, que nous serons heureux de recevoir.

- D'autre part, une base de données sur les images publiées dans Mappemonde depuis le tout premier numéro et jusqu'au numéro 123 inclus. Cette base, construite par Hervé Théry et Clarisse Didelon Loiseau, référence 6470 "images »: des cartes en tous genres, animées ou non, mais également des photographies, des graphiques, des chorèmes, des tableaux, des dessins, des images satellites, etc., bref, tout type d'image qui fait l'objet de l'analyse géographique ou est utilisée dans la démonstration scientifique. Ces images sont décrites selon l'échelle représentée, le thème traité, le type de représentation et permettent de saisir, par exemple, l'irruption d'une innovation dans les méthodes mises en œuvre en cartographie, ou de dresser le tableau des mutations de la géographie en tant que discipline 
autant que de la société dans son ensemble, avec la place de plus en plus importante accordée aux outils numériques.

Ce numéro des « 30 ans » a également donné lieu à un exercice pour le moins original. Nous avons identifié des articles présentant un caractère "prospectif ", publiés au cours des années dans Mappemonde et avons contacté les auteurs, afin de leur demander de faire un retour sur ces textes. Sur la petite vingtaine d'articles identifiés, sept auteurs nous ont répondu, souvent avec enthousiasme et ont rapidement produit le texte réflexif que nous leur avions commandé. La première livraison de ce numéro spécial vous présente donc un retour sur des articles prospectifs consacrés à Nantes (D. Rapetti), Bordeaux (M. Banzo), l'île d'Yeu (P. Pottier et M. Robin) ou le tennis dans le monde (P. Dumolard).

D'autres contributions sont à venir... de la part des membres du comité comme d'auteurs et nous invitons nos lecteurs à s'approprier notre base de données s'ils le souhaitent pour produire leur propre analyse.

Aujourd'hui, alors que toutes les revues ont une édition en ligne, la publication d'images n'est plus l'apanage de Mappemonde. Elle pourrait perdre sa spécificité, son identité, mais... au-delà des changements évoqués, l'âme de Mappemonde reste la même, celle d'une revue scientifique ouverte dédiée à l'image et en particulier l'image en géographie. Son identité reste forte, et la qualité du travail d'évaluation et d'accompagnement, d'édition scientifique et cartographique reste notre motivation, c'est cela que reconnaissent les auteurs qui nous proposent leurs contributions, et les lecteurs qui nous suivent.

Pour terminer, nous remercions l'équipe de production (Laetitia Faure et Mathieu Coulon), le CNRS pour son soutien (notamment l'UMR ESPACE et l'Institut des SHS), mais surtout les auteurs et coordinateurs de numéros qui nous font confiance à la fois pour l'évaluation et la diffusion de leurs articles et enfin, ceux pour qui nous travaillons avant tout et à qui nous devons tant : nos lecteurs.

\section{INDEX}

Thèmes : 30 ans de publication dans Mappemonde

\section{AUTEURS}

\section{CLARISSE DIDELON-LOISEAU}

Université Paris 1

\section{LAURENT JÉGOU}

Université de Toulouse-Jean Jaurès, UMR LISST-Cieu 